\title{
329 - A psychological needs-based intervention to reduce depression in newly admitted nursing home residents
}

Authors: Tanya E. Davison, Marita P. McCabe, Lucy Busija, \& Annette Graham

Background: Depression is common in nursing homes, with newly admitted residents at a particularly high risk. Current prevention and treatment approaches have failed to impact on the high rates of depression and new approaches are required. We have developed a novel intervention that addresses individual psychological needs in newly admitted residents, which is implemented in collaboration with residents and facility staff. The Program to Enhance Adjustment to Residential Living (PEARL) is a 5session intervention based on Self-Determination Theory that aims to tailor care to enhance residents' autonomy, competence and relations.

Methods: A cluster randomised controlled trial was conducted to determine the effectiveness of PEARL in reducing depression in newly admitted nursing home residents, compared to a treatment as usual control condition. A total of 216 residents with normal cognition or mild-moderate cognitive impairment, living in 42 nursing homes in Melbourne, Australia, participated in the study. The sample included 76 men and 140 women, aged $62-99$ years $(M=85.5, S D=7.3)$. The primary outcome was level of depressive symptoms, assessed using the Cornell Scale for Depression in Dementia at baseline and at 8, 16 (primary endpoint), and 31 weeks follow-up. Multi-level modelling, accounting for within-facility clustering and repeated assessments, was used to determine the effectiveness of the intervention, employing an intention-to-treat approach.

Results: At 16-weeks, fewer symptoms of depression were observed among those in the intervention group $(M=7.0, S D=5.6)$ than in the control group $(M=8.6, S D=6.5)$. Multi-level modelling of unadjusted data showed a significant condition (intervention, control) by time $(T 1, T 2, T 3)$ interaction ( $p$ $=.021$ ), indicating that the PEARL intervention led to a reduction in the occurrence of depressive symptoms. The treatment effect was maintained at 31 weeks follow-up $(p=.004)$.

Discussion: This study provides evidence that an intervention designed to address newly admitted residents' individual psychological needs reduced symptoms of depression in subsequent months. This brief, simple intervention may be suitable for broad implementation across long-term care settings. However, future research to determine if the intervention can be implemented by trained facility staff in situ is warranted. 